\title{
O Envolvimento da Atenção na Codificação da Informação Visuoespacial Integrada
}

\author{
Jeanny Joana Rodrigues Alves de Santana \\ César Galera² \\ Universidade de São Paulo
}

\begin{abstract}
RESUMO - O objetivo deste estudo foi investigar o envolvimento de recursos da atenção na codificação e manutenção da informação visual e espacial na memória de trabalho. Utilizou-se um paradigma de tarefas duplas em que uma tarefa primária de localização espacial foi realizada simultaneamente a uma tarefa atentiva secundária de discriminação de tons. O desempenho dos participantes $(\mathrm{n}=20)$ na tarefa primária foi afetado pela presença e pela similaridade entre os tons da tarefa secundária, e também, pela instrução de priorizar uma ou outra tarefa. Os resultados indicam que recursos atentivos (do executivo central) estão envolvidos na codificação e na manutenção ativa da informação integrada na memória visuoespacial, assim como na manutenção dos objetivos das tarefas a serem realizadas simultaneamente.
\end{abstract}

Palavras-chave: memória de trabalho; memória visuoespacial; conjunção visuoespacial; executivo central.

\section{The Involvement of Attention in the Encoding of Integrated Visuo-spatial Information}

\begin{abstract}
The objective of this study was to investigate the involvement of attention in the encoding and maintenance of information in visual and spatial working memory. We used a dual task paradigm in which a primary task of spatial localization was performed simultaneously with a secondary attentive task of tone discrimination. The participants' performance $(n=20)$ in the primary task was affected by the presence and similarity between the tones of the secondary task, and also the instruction to prioritize one task or another. The results indicate that attentive resources (central executive) are involved in encoding and active maintenance of integrated information in visuo-spatial memory, as well as in maintaining the objectives of the tasks to be performed simultaneously.
\end{abstract}

Keywords: working memory; visuo-spatial memory; visuo-spatial binding; central executive.

A memória de trabalho é um sistema de armazenamento e manipulação de informações que nos permite guardar uma informação em curto prazo enquanto realizamos tarefas que exigem raciocínio, aprendizagem e compreensão. Este sistema abrange um conjunto de componentes de armazenamento coordenados por um sistema atentivo denominado executivo central. Um dos componentes de armazenamento - o laço fonológico - é dedicado à informação verbal, e o outro, dedicado à informação visuoespacial, é responsável por armazenar a aparência e as relações espaciais entre os objetos. Finalmente, o buffer episódico é o componente responsável por armazenar e integrar informações das duas modalidades entre si e com a memória de longo prazo (Baddeley, 2007; Repovš \& Baddeley, 2006).

O laço fonológico compreende um sistema de armazenamento temporário e um sistema de recitação verbal-auditivo, semelhante à fala subvocal (Repovš \& Baddeley, 2006). A informação verbal tem acesso automático ao laço fonológico

1 Apoio: CNPQ - Conselho Nacional de Desenvolvimento Científico e Tecnológico e da FAPESP - Fundação de Amparo à Pesquisa do Estado de São Paulo. Agradecimentos à R. Oliveira (IC - CNPQ)

2 Endereço de correspondência: - Universidade de São Paulo, Faculdade de Filosofia, Ciências e Letras de Ribeirão Preto- SP, Departamento de Psicologia, Av. Bandeirantes, 3900 bloco 6, sala 34 - Bairro Monte Alegre .Ribeirão Preto, SP, CEP 14040-901. E-mail: algalera@usp.com através da nomeação dos estímulos, mas o conteúdo verbal advindo dos outras modalidades, por exemplo, da leitura, entra no sistema pela recodificação fonológica (nomeação). Dado este processo, a informação é armazenada, porém, ainda sujeita à interferência de outras informações ou ao decaimento do conteúdo por causa da passagem do tempo. O material fonológico pode ser recitado por um processo de articulação subvocal que permite a manutenção da informação fonológica por mais tempo (Henson, Burgess \& Frith, 2000). Logie (1995) supôs que um processo semelhante ocorre para a modalidade visuoespacial. Segundo ele, existe um armazenador passivo denominado cache visual e um mecanismo ativo de recitação chamado de inner scribe. O primeiro estaria dedicado ao armazenamento da informação visual estática e o segundo se relacionaria ao processo de recitação do conteúdo visual, através de processos de movimento.

De acordo com Baddeley (2002), o executivo central teria a função de coordenar as informações advindas dos outros subsistemas, ou seja, focar a atenção, dividi-la, deslocá-la e de realizar o intercâmbio entre memória de trabalho e memória de longo prazo. Esta relação é estabelecida, por exemplo, nas operações de ativação de esquemas cognitivos pré-existentes (estratégias) para realização de tarefas complexas (Norman \& Shallice, 1986; Ang \& Lee, 2010). As funções executivas estão relacionadas com a ativação de redes neurais que envolvem tanto regiões do lobo frontal como do lobo parietal 
(Collette \& Van der Linden, 2002). Neste sentido, além dos recursos cognitivos específicos subjacentes à memória visuoespacial e verbal, recursos cognitivos gerais também estão envolvidos com a retenção das informações. Neste estudo investigamos o envolvimento da atenção no armazenamento da informação visuoespacial numa tarefa de localização espacial (Galera \& Fuhs, 2003; Walker, Hitch \& Duroe, 1993).

A hipótese do envolvimento de recursos gerais da atenção gerenciados pelo executivo central no armazenamento das informações de modalidades específicas tem sido investigada através do paradigma das tarefas duplas. Nesse paradigma avalia-se o desempenho em uma tarefa primária (visuoespacial ou verbal) realizada simultaneamente a uma tarefa secundária que exige recursos da atenção. Se a tarefa secundária prejudica o desempenho da tarefa principal (de qualquer modalidade de informação), pode-se concluir pela utilização de recursos gerais da atenção para armazenamento da informação na memória de trabalho (Klauer \& Zhao, 2004). Por exemplo, Salway e Logie (1995) investigaram o envolvimento de recursos atentivos gerais na memória para a tarefa de Brooks (1967) realizada com base na matriz visuoespacial e na recitação verbal. Essas tarefas foram realizadas isoladamente, ou juntamente com três tarefas secundárias: a supressão articulatória, a supressão espacial, e a geração de dígitos aleatórios. Quando as tarefas de Brooks foram realizadas isoladamente, os resultados sugerem que as duas versões utilizam recursos cognitivos diferentes, especializados, envolvidos na geração da ação. No entanto, quando a geração de dígitos aleatórios foi muito exigente em termos atentivos, tanto o desempenho na versão espacial como na versão verbal da tarefa de Brooks foram prejudicados, sugerindo que essas tarefas dependem fortemente de recursos atentivos gerais, além dos recursos especializados dos sistemas diretamente envolvidos.

Muitos outros estudos têm mostrado que o desempenho da memória espacial é afetado por tarefas centradas em recursos cognitivos gerais tais como a geração de letras e dígitos aleatórios (Fisk \& Sharp, 2003; Rudkin, Pearson \& Logie, 2007), ou a contagem regressiva de três em três (Galera \& Fuhs, 2003). Klauer e Stegmaier (1997) também mostraram que a realização de uma tarefa de discriminação de tons durante o intervalo de retenção na tarefa de blocos de Corsi prejudica a recordação da informação espacial. Isso acontece mesmo quando os tons vêm da mesma posição espacial, sugerindo que os efeitos das tarefas secundárias de discriminação de tons não se devem à interferência espacial específica da tarefa secundária, mas ao envolvimento do executivo central no processo de recitação na memória espacial serial.

Os estudos acima mostram o papel do executivo central na manutenção da informação espacial codificada isoladamente, em tarefas semelhantes à dos blocos de Corsi. Entretanto, ainda não está esclarecido o envolvimento de recursos executivos no armazenamento integrado da informação visual e espacial na memória de trabalho. Estudos recentes têm sugerido que o armazenamento integrado da informação visual e espacial não demanda recursos gerais da atenção e, além disso, indicam que a localização espacial é codificada de maneira não intencional (incidentalmente) em tarefas de reconhecimento visual (Maybery et al., 2009; Olson \& Marshuetz, 2005). Ou seja, a codificação incidental da informação espacial juntamente com a informação visual sugere que ambas são codificadas automaticamente, sem que haja a necessidade de envolvimento de recursos do executivo central nesse processo (ver também Jiang, Olson \& Chun, 2000; Olson \& Marshuetz, 2005). Neste estudo investigamos o envolvimento dos recursos atentivos na codificação da forma visual associada à localização espacial através do efeito de uma tarefa secundária de discriminação de tons. $\mathrm{O}$ envolvimento dos recursos atentivos na codificação de forma e localização foi avaliado através do efeito de uma tarefa secundária de discriminação de tons sobre o desempenho numa tarefa de localização espacial.

Na tarefa de localização espacial (Galera \& Fuhs, 2003; Walker, Hitch \& Duroe, 1993) o participante deve memorizar quatro estímulos visuais apresentados em ordem temporal e espacial aleatória na tela de um monitor e, depois de um intervalo de retenção, deve dizer onde foi originalmente apresentado um estímulo teste. A realização desta tarefa exige que a localização e identidade de quatro eventos sejam codificadas e armazenadas de forma integrada, até que seja apresentado o estímulo teste. Esta codificação integrada da informação visual e da localização espacial, necessária na tarefa de localização espacial, demanda recursos atentivos? Para responder a essa questão avaliamos o efeito de uma tarefa secundária de discriminação de tons sobre o desempenho numa tarefa de localização espacial.

A tarefa de discriminação de tons que utilizamos é inspirada na tarefa de contagem de tons utilizada frequentemente em estudos sobre aprendizagem implícita de sequências. Esta tarefa afeta a aprendizagem de sequências de estímulos apresentados em diferentes posições. As evidências sugerem que esse prejuízo pode ser provocado pela redução da atenção disponível para a aprendizagem da sequência (Curran \& Keele, 1993; Nissen \& Bullemer, 1987) embora essa sugestão esteja sob disputa (Stadler, 1995; Frensch, Lin \& Buchner, 1998). Curran e Keele (1993) sugerem que a aprendizagem implícita de sequências pode depender de recursos atentivos, uma vez que a tarefa secundária de contagem de tons afeta $\mathrm{o}$ desempenho em tarefas de memória para eventos apresentados sequencialmente e em diferentes posições espaciais da tela do computador. Por outro lado, Frensch, Lin e Buchner (1998) sugerem que a atenção necessária para a tarefa de contagem de tons afetaria a organização da resposta, interferindo na expressão do que foi implicitamente aprendido, ao invés de afetar a aprendizagem desta sequência. De toda a forma, exceto pela disputa sobre o lócus de ação da contagem de tons, que não é tema deste texto, os estudos acima consideram que a tarefa de contar tons demanda recursos atentivos.

No presente estudo nossa suposição foi que a tarefa secundária deveria prejudicar a codificação integrada da identidade visual e da localização e, consequentemente, a memória visuoespacial, na medida em que a discriminação de tons utiliza recursos atentivos necessários também à codificação da informação na tarefa de localização espacial, como verificado nos estudos de Curran e Keele (1993) e de Nissen e Bullemer (1987). Também consideramos que a dificuldade da tarefa de discriminação, manipulada através da similaridade entre os estímulos a serem discriminados, deve contribuir para a interferência a ser provocada pela tarefa secundária. Além disso, resultados de um estudo 
piloto haviam sugerido que os participantes, quando instruídos a realizar as duas tarefas simultaneamente tendem a priorizar ora uma, ora outra tarefa. Em uma tentativa de ter um controle maior sobre a estratégia de realização na tarefa dupla, os sujeitos realizaram duas sessões (com intervalo de 15 minutos entre elas, em média), nas quais foram instruídos a priorizar a tarefa de discriminação de tons ou a tarefa de localização espacial. Os procedimentos adotados neste estudo estão de acordo com as diretrizes e normas da resolução de ética na pesquisa.

\section{Método}

\section{Delineamento}

O tipo de tarefa foi um fator manipulado inter participantes, ou seja, todos eles realizaram as tarefas de reconhecimento da localização espacial e de discriminação de tons. A posição serial do estímulo teste também foi um fator manipulado inter participantes, ou seja, a probabilidade do estímulo teste corresponder a uma das posições seriais memorizadas foi igualmente distribuída, para todos os participantes. A ordem de instrução de prioridade foi um fator manipulado entre participantes. Um grupo deveria priorizar na primeira sessão a tarefa de discriminação de tons e na segunda sessão a tarefa de localização espacial (Grupo 1) e o segundo grupo (Grupo 2) a ordem contrária. A ordem de realização das provas segundo a similaridade dos tons também foi um fator manipulado entre participantes. Para metade dos sujeitos, o bloco inicial foi realizado com tons de similaridade alta e o bloco final com tons de similaridade baixa. Para a outra metade, ocorreu o inverso.

\section{Participantes}

Vinte universitários com idade variando de 18 a 40 anos, todos com visão normal ou corrigida foram voluntários em duas sessões experimentais.

\section{Material e Estímulos}

Os estímulos visuais eram padrões criados pelo preenchimento semi-aleatório de 8 células de uma matriz 4 x 4 com $4 \mathrm{~cm}$ de lado, apresentados em quatro localizações na tela de um monitor. As localizações nas quais os estímulos foram apresentados eram marcadas por quatro molduras quadradas, com $4 \mathrm{~cm}$ de lado, definidas apenas por contorno preto, dispostas na horizontal, no centro da tela do monitor. A distância entre duas molduras vizinhas era de $2 \mathrm{~cm}$. O estímulo teste era apresentado em uma moldura com as mesmas proporções, situado no centro da tela, $4 \mathrm{~cm}$ abaixo das posições onde eram apresentados os estímulos (Figura 1). Os estímulos sonoros da tarefa de discriminação foram tons puros com frequências de $1000 \mathrm{~Hz}, 1500 \mathrm{~Hz}$ e $2000 \mathrm{~Hz}$, cada um com duração de $50 \mathrm{~ms}$. O experimento foi realizado com o utilitário E-prime v. 1.2 (Schneider, Eschman \& Zuccolotto, 2002).

\section{Procedimento}

Foi utilizado um paradigma de tarefas duplas no qual uma tarefa de localização espacial era a tarefa primária e uma tarefa de discriminação de tons, a tarefa secundária. A tarefa de localização espacial consistiu na apresentação sequencial de quatro estímulos em quatro posições predefinidas na tela do monitor, seguidos por um estímulo teste apresentado em uma posição neutra. A tarefa do participante foi identificar a posição espacial na qual o estímulo teste foi originalmente apresentado. Cada prova desta tarefa tinha início com a apresentação das molduras, que permaneciam na tela durante toda a prova. Depois de $750 \mathrm{~ms}$ tinha início a apresentação dos estímulos nas diferentes posições espaciais. A ordem na qual os estímulos foram apresentados nas diferentes posições foi semi-aleatória de forma a que a localização espacial e a posição serial do estímulo teste na sequência memorizada $\left(1^{\circ}\right.$, $2^{\circ}, 3^{\circ}$ ou $4^{\circ}$ estímulo) fossem equiprováveis. Cada estímulo da seqüência foi apresentado para memorização durante $750 \mathrm{~ms}$, com um intervalo de $750 \mathrm{~ms}$ entre cada estímulo. O estímulo teste era apresentado $750 \mathrm{~ms}$ após a apresentação do último estímulo da sequência e permanecia na tela até que o participante emitisse sua resposta sobre a posição na qual esse estímulo havia sido apresentado. O participante deu sua resposta no teclado do computador, pressionando as teclas $\mathrm{S}$ (teste na posição à esquerda da tela), F (teste na posição centro esquerda), com os dedos anular e indicador da mão esquerda, e as teclas $\mathrm{J}$ (teste na posição centro direita) e L (teste na posição à direita) com os dedos indicador e anular da mão direita, respectivamente. Para iniciar a prova seguinte, o participante deveria pressionar a barra de espaço. Dois segundos depois, tinha início uma nova prova.

$\mathrm{Na}$ tarefa de discriminação auditiva, o sujeito deveria contar o número de tons agudos apresentados em uma sequência de quatro tons graves e agudos. A duração de cada tom foi de $50 \mathrm{~ms}$, com intervalo de $1450 \mathrm{~ms}$ entre eles, de forma que quando as duas tarefas fossem realizadas simultaneamente a apresentação do tom coincidisse com a apresentação do estímulo visual. O participante deveria contar quantos tons agudos haviam sido apresentados na sequência $(0,1,2$ ou $3)$. Os estímulos da tarefa de discriminação poderiam ser de similaridade baixa, com tons de $1000 \mathrm{~Hz}$ e $2000 \mathrm{~Hz}$, ou de similaridade alta, com tons de $1000 \mathrm{~Hz}$ e $1500 \mathrm{~Hz}$. A resposta era dada no teclado numérico ao final da sequência. Quando esta tarefa foi realizada isoladamente, a tela do monitor permanecia em branco enquanto os tons eram apresentados. Quando esta tarefa era realizada juntamente com a tarefa de localização os tons eram apresentados simultaneamente com cada estímulo visual (Figura 1).

Nas provas em que as duas tarefas eram realizadas em conjunto, a apresentação dos estímulos visuais e auditivos tinha início simultaneamente. Nessas provas, logo após a apresentação do último estímulo a ser memorizado o participante era instruído por uma mensagem na tela a dar sua resposta à tarefa de discriminação de tons, indicando no teclado numérico quantos estímulos agudos haviam sido 


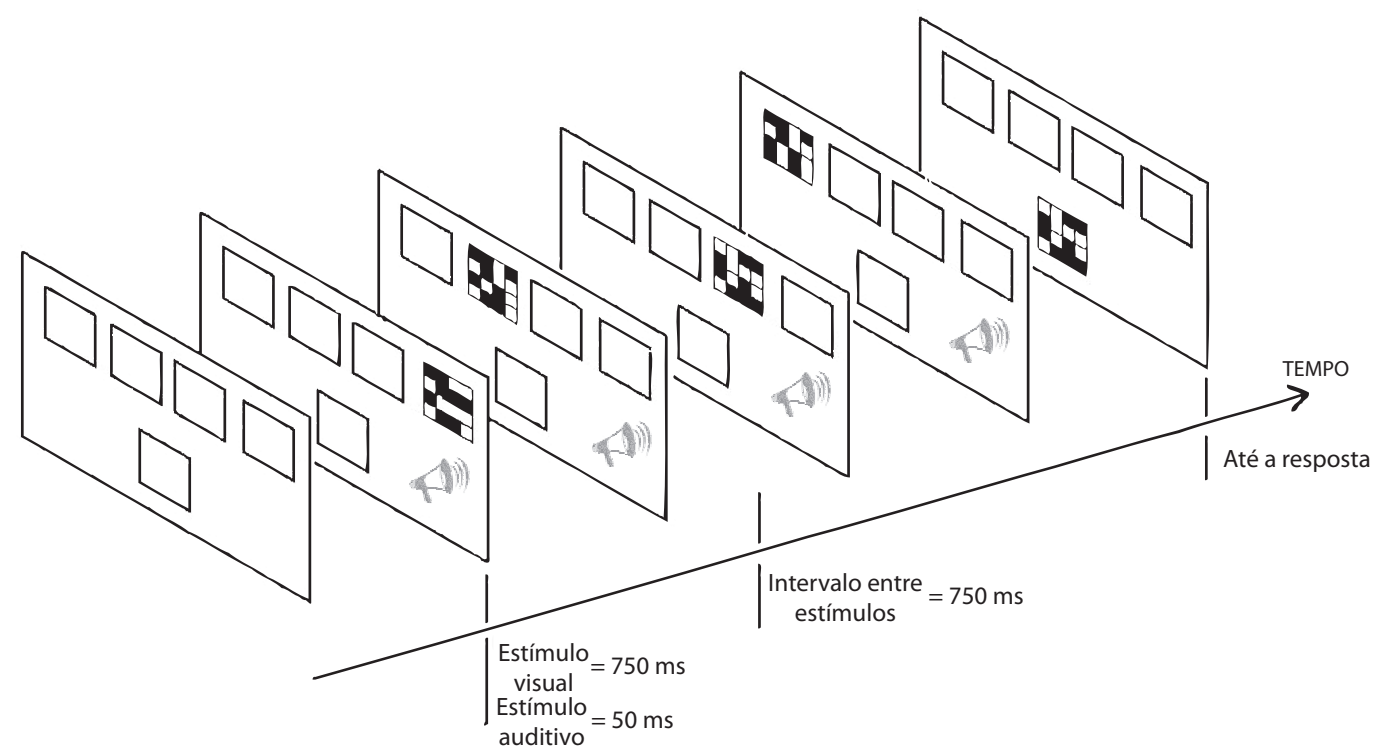

Figura 1. Sequência de eventos de uma prova experimental. A tarefa de memória visuoespacial é representada pela sequência de matrizes e a tarefa de discriminação de tons é representada pelo desenho em cinza.

apresentados. Em seguida, eram instruídos a informar a localização na qual o estímulo teste da tarefa de localização havia sido apresentado. Para passar de uma prova para outra, o participante deveria pressionar a barra de espaço após a emissão da resposta da tarefa de localização. Nas provas em que as tarefas foram realizadas isoladamente as instruções para a resposta eram específicas às tarefas realizadas.

Foram realizadas duas sessões experimentais. Na primeira sessão, nas provas em que ambas as tarefas eram realizadas simultaneamente, metade dos participantes foi instruído a priorizar a realização da tarefa de localização. A outra metade dos participantes foi instruída a priorizar a tarefa de discriminação de tons. Na segunda sessão a instrução de prioridade foi invertida. Embora fossem instruídos a priorizar uma ou outra tarefa, os participantes também foram instruídos a realizar as duas tarefas da forma mais acurada o possível.

Em cada sessão foram realizadas 140 provas válidas distribuídas em quatro blocos de provas. Em um bloco foram realizadas 40 provas apenas com a tarefa de localização visuoespacial. Em outro bloco, foram realizadas 20 provas apenas com a tarefa de discriminação de tons (neste bloco, em metade das provas os tons eram de similaridade baixa e de similaridade alta na outra metade). Em outros dois blocos, com 40 provas cada um, as tarefas de localização visuoespacial e discriminação foram executadas simultaneamente. Em um dos blocos em que as tarefas foram realizadas simultaneamente os tons eram de similaridade baixa e no outro os tons eram de similaridade alta. No início de cada bloco experimental cada participante passava por 14 provas de treino. A ordem de apresentação dos blocos foi aleatorizada considerando 3 blocos: tarefa de localização espacial, tarefa de discriminação de tons e tarefas executadas simultaneamente.

O envolvimento de recursos atentivos na tarefa de localização espacial foi investigado através de um paradigma de tarefas duplas no qual a tarefa de localização espacial foi realizada simultaneamente a uma tarefa secundária de discriminação de tons. O delineamento experimental envolveu medidas repetidas no desempenho na tarefa localização em função dos fatores: instrução da prioridade (tarefa de localização, tarefa de discriminação de tons), presença da tarefa secundária (presente com similaridade alta, presente com similaridade baixa e ausente) e posição serial do estímulo teste na tarefa de localização espacial $\left(1^{\circ}, 2^{\circ}, 3^{\circ}\right.$ e $4^{\circ}$ estímulo apresentado). A ordem pela qual os participantes passaram pela instrução de prioridade (primeira ou segunda sessão) foi um fator manipulado entre sujeitos.

\section{Resultados}

O objetivo deste estudo foi avaliar o envolvimento dos recursos atentivos na codificação de forma e localização na tarefa de localização espacial através do efeito de uma tarefa secundária de discriminação de tons. A suposição inicial era que um prejuízo na tarefa primária causado pela realização simultânea da tarefa secundária permitiria concluir que a conjunção visuoespacial requer recursos gerais da atenção para ser codificada na memória de trabalho.

\section{Tarefa de localização espacial}

A porcentagem de respostas corretas na tarefa de localização espacial foi submetida a uma análise de variância considerando os seguintes fatores: instrução de prioridade (tarefa de localização espacial, tarefa de discriminação de tons), presença da tarefa secundária de discriminação de tons (presente com tons de similaridade alta, presente com tons de similaridade baixa e ausente), e posição serial do estímulo teste da tarefa de localização espacial (primeiro, segundo, terceiro e quarto estímulo apresentado). A ordem pela qual os sujeitos passaram pelas diferentes prioridades foi considerada como fator entre grupos. 
No geral, a ordem pela qual os dois grupos passaram pelas condições não teve um efeito significativo sobre a média (M) de acertos $[F(1,18)=0,04 ; p=0,851]$. O desempenho na tarefa de localização foi afetado pela instrução de prioridade. Nas provas em que a prioridade era a tarefa de localização, a taxa de respostas corretas foi em média $62 \%(\mathrm{EPM}=5 \%)$ e naquelas em que a prioridade foi a discriminação de tons, os acertos foram em média 55\% $(\mathrm{EPM}=5 \%)[\mathrm{F}(1,18)=12,09$; $\left.p=0,003 ; \eta_{p}^{2}=0,4\right]$. Isto sugere que recursos atentivos foram divididos entre as duas tarefas, ocasionando um prejuízo na tarefa de localização espacial quando realizada na condição em que a prioridade era a discriminação. O desempenho foi afetado pela presença da tarefa de discriminação $[\mathrm{F}(2,36)=12,73$; $\mathrm{p}<$ 0,$\left.001 ; \eta_{\mathrm{P}}^{2}=0,52\right]$. Uma análise post hoc (Newman-Keuls, $\mathrm{p}<$ 0,05 ) confirma que o desempenho foi melhor quando a tarefa de localização foi realizada sem a tarefa de discriminação de tons $(\mathrm{M}=63 \%, \mathrm{EPM}=5 \%) \mathrm{e}$, quando as duas tarefas eram realizadas simultaneamente, o desempenho foi melhor quando os tons tinham similaridade baixa $(\mathrm{M}=59 \%, \mathrm{EPM}=6 \%)$ do que quando tinham similaridade alta $(M=54 \%, E P M=6 \%)$.

De maneira geral, o efeito da posição serial do estímulo teste na tarefa de localização mostra um forte efeito de recência $\left[F(3,54)=105,82 ; p<0,001 ; \eta_{p}^{2}=0,94\right]$. O desempenho é melhor quando o estímulo teste é o último estímulo memorizado $(\mathrm{M}=86 \%$; EPM $=3 \%)$ e se mantém estável para as três primeiras posições $(\mathrm{M}=49 \%, 47 \%$, e $52 \%$, respectivamente).

A ordem pela qual os sujeitos passaram nas duas sessões interagiu de forma significativa com o desempenho na tarefa priorizada $\left[\mathrm{F}(1,18)=10,92 ; \mathrm{p}=0,004 ; \eta_{\mathrm{P}}^{2}=0,38\right]$. De acordo com esta interação os participantes que foram instruídos a priorizar a tarefa de discriminação de tons na primeira sessão tiveram uma melhora substancial no desempenho na segunda sessão, quando foram instruídos a priorizar a tarefa de localização. $\mathrm{O}$ desempenho desses sujeitos saltou de $\mathrm{M}=52 \%(\mathrm{EPM}=8 \%)$ na primeira sessão para $\mathrm{M}=64 \%(\mathrm{EPM}=8 \%)$ na segunda. Já o desempenho dos participantes que foram instruídos a priorizar a tarefa de localização na primeira sessão se manteve estável na segunda sessão, quando deveriam priorizar a tarefa de discriminação. Nesse caso o desempenho foi de $\mathrm{M}=58 \%$ (EPM $=8 \%$ ) na primeira sessão (prioridade na localização) para $\mathrm{M}=$ $59 \%(\mathrm{EPM}=8 \%)$ na segunda sessão (prioridade na tarefa de discriminação). Esta interação sugere que o desempenho na tarefa de localização não é explicado pela predominância de processamento de uma ou outra modalidade do estímulo. Se fosse este o caso, a prioridade em uma ou outra tarefa repercutiria de maneira constante no desempenho, independente da ordem de realização. O desempenho é melhor explicado pela combinação entre tipo de instrução e ordem de execução. Mais precisamente, a realização inicial da tarefa de localização com prioridade na discriminação de tons envolveu a criação de uma regra específica de execução, que pode ter facilitado a realização posterior da tarefa de localização com prioridade na localização.

Além destes efeitos gerais, houve uma interação entre a similaridade dos tons e a instrução de prioridade dada aos sujeitos $\left[\mathrm{F}(2,36)=3,26 ; \mathrm{p}=0,05 ; \eta_{\mathrm{p}}^{2}=0,28\right]$. Quando os sujeitos foram instruídos a dar prioridade à tarefa de localização, o desempenho foi melhor quando os tons eram de similaridade baixa $(\mathrm{M}=65 \%$ de acertos; $\mathrm{EPM}=6 \%)$ do que na similaridade alta $(\mathrm{M}=58 \% ; \mathrm{EPM}=6 \%)$, ou quando os tons não estavam presentes $(\mathrm{M}=65 \% ; \mathrm{EPM}=5 \%)$. Na prioridade do som, o desempenho para a localização foi em média $53 \%(\mathrm{EPM}=6 \%)$ quando a similaridade era baixa e média de $51 \%(\mathrm{EPM}=6 \%)$ quando a similaridade era alta. Juntos, estes resultados mostraram que recursos atentivos foram divididos entre as tarefas e, quando a discriminação de tons demandou maior carga atentiva, ou seja, foi mais difícil porque a similaridade entre os tons era alta, ocorreu um maior prejuízo na tarefa de memória.

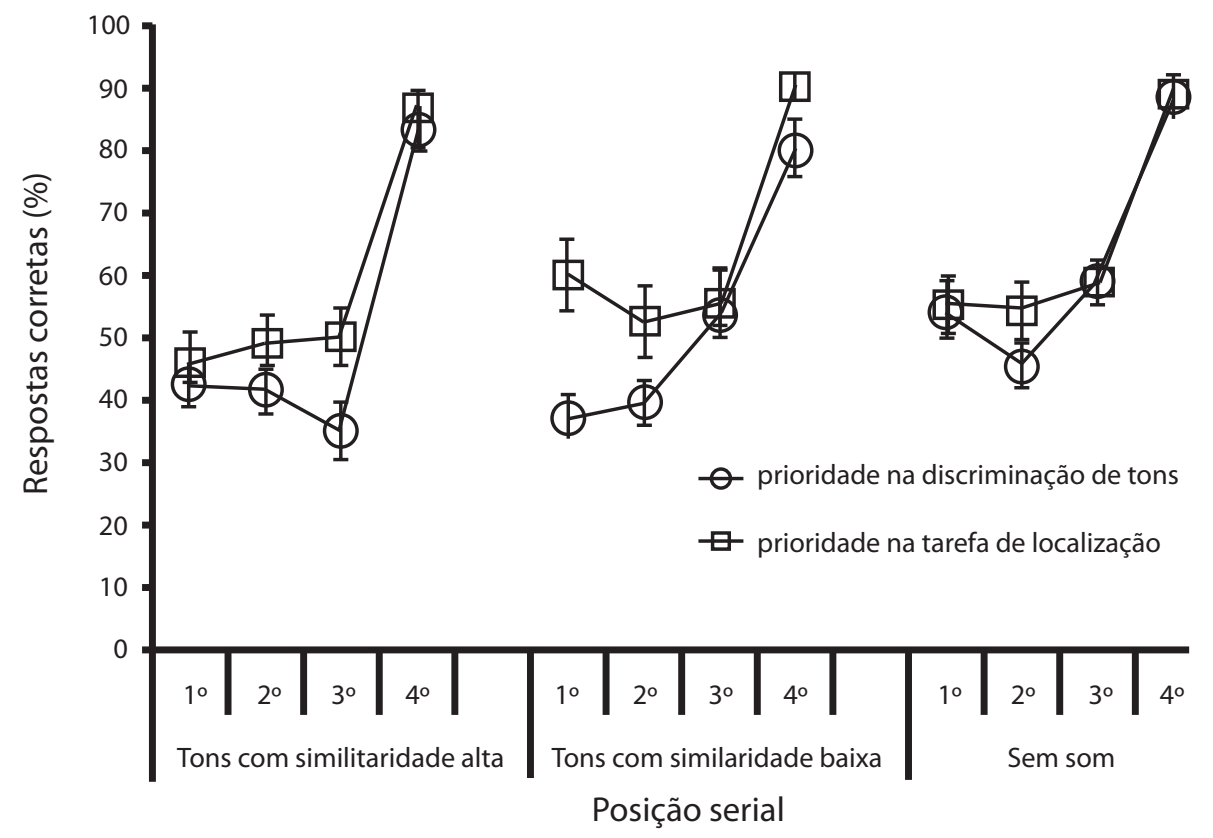

Figura 2. Porcentagem de respostas corretas para localização visuoespacial em função da posição do estímulo teste $\left(1^{\circ}, 2^{\circ}, 3^{\circ}\right.$ e $\left.4^{\circ}\right)$ e das condições experimentais: tarefa atentiva com alta similaridade entre tons; tarefa atentiva com baixa similaridade entre tons e sem tarefa atentiva, combinadas com prioridade estipulada pelo experimentador na tarefa de localização visuoespacial ou na tarefa atentiva. 
A interação entre similaridade dos tons, instrução de prioridade e posição serial $\left[F(6,108)=2,60 ; p=0,022 ; \eta_{P}^{2}\right.$ $=0,78]$ (Figura 2) indica que o desempenho da memória para localização do primeiro estímulo apresentado é $17 \%$ superior na condição em que a similaridade dos tons é baixa e a prioridade é a tarefa de localização, que na condição em que a similaridade dos tons é alta e a prioridade é a tarefa de discriminação. De maneira semelhante, o desempenho da memória para localização do último estímulo memorizado é $6 \%$ melhor nas provas em que os tons eram de similaridade baixa e a prioridade era a localização, do que nas provas em que os tons eram muito similares e a prioridade era a tarefa de discriminação. Existe ainda uma inversão na tendência do efeito da instrução quando se trata do terceiro estímulo apresentado: neste caso, a instrução para priorizar a tarefa visual provoca uma melhora pequena $(2 \%)$ na recordação quando os estímulos têm similaridade baixa e um aumento substancial (15\%) quando os estímulos têm similaridade alta.

\section{Tarefa de discriminação de tons}

A média do desempenho dos sujeitos na tarefa de discriminação de tons quando realizada sozinha é de $97,7 \%$ $(\mathrm{EPM}=1 \%)$, sendo prejudicada quando a prova é realizada juntamente com a localização visuoespacial ( $\mathrm{M}=75 \%$; EPM $=4 \%)\left[\mathrm{F}(1,18)=97,85 ; \mathrm{p}<0,001 ; \eta_{\mathrm{P}}^{2}=0,85\right]$. Quando realizadas simultaneamente, a prioridade à tarefa visuoespacial prejudica o desempenho na tarefa de discriminação $(\mathrm{M}=81,0 \% ; \mathrm{EPM}=5 \%)$ em comparação quando a tarefa de discriminação deve ser priorizada $(\mathrm{M}=91,9 \% ; \mathrm{EPM}=2 \%)$ $\left[\mathrm{F}(1,18)=23,01 ; \mathrm{p}<0,001 ; \eta_{\mathrm{P}}^{2}=0,56\right]$. Este dado confirma que os recursos atentivos realmente foram divididos entre as tarefas, dificultando o desempenho da discriminação de tons quando esta tarefa foi realizada concomitantemente à tarefa de memória.

Os participantes tiveram dificuldade em desempenhar a tarefa de discriminação quando a similaridade dos tons era alta e realizada juntamente com a tarefa visuoespacial $(\mathrm{M}=$ $71 \%$; EPM $=4 \%$ ) em comparação à condição em que a resposta era dada para tons de similaridade baixa apresentados sem a tarefa visuoespacial $(\mathrm{M}=96 \% ; \mathrm{EPM}=1 \%)[\mathrm{F}(1,18)$ $=18,573 ; \mathrm{p}<0,001]$.

\section{Discussão}

Neste estudo investigamos o envolvimento de recursos do executivo central na codificação da informação visuoespacial na memória de trabalho. Nossos dados mostraram que a memória de trabalho utiliza recursos gerais da atenção para codificar esta informação, ou seja, o desempenho na tarefa de memória depende tanto de recursos específicos do esboço visuoespacial quanto de recursos gerais do executivo central.

A interação de recursos específicos (do esboço visuoespacial) com recursos gerais (do executivo central) para o registro de informações na memória confirma o caráter ativo inerente à memória de trabalho. Estudos prévios confirmam que para criar e manter uma representação na memória suficientemente ativa para guiar o comportamento para um objetivo é preciso que uma rede neural complexa (envolvida com funções executivas e de armazenamento) seja ativada (D’Esposito, 2007).

Nossos dados estão de acordo com estudos anteriores que mostraram a dependência do esboço visuoespacial em relação ao executivo central. Há uma forte correlação entre processos executivos e funções de visualização espacial e de processamento de relações espaciais (Miyake, Friedman, Rettinger, Shah \& Hegarty, 2001). Nossos resultados mostram que o executivo central, além de estar envolvido no armazenamento espacial (Klauer \& Stegmaier, 1997) está também envolvido na codificação da informação visuoespacial.

O envolvimento do executivo central na codificação da informação visuoespacial está relacionado com a coordenação de processos de nível inferior (informação sensorial) e superior (processos motores da resposta, por exemplo) em função de um objetivo da tarefa. Uma vez estabelecida uma representação interna do objetivo, mecanismos de controle direcionam a codificação da informação (Miller \& Cohen, 2001). Se há uma falha em manter a orientação para o objetivo da tarefa, há prejuízo na execução da resposta (Szmalec, 2005), mesmo que o armazenador específico àquela modalidade do estímulo não esteja sob efeitos específicos de interferência, como foi evidenciado pelo fato de que o desempenho da memória visuoespacial foi pior quando os tons da tarefa atentiva eram de similaridade alta (tons mais complexos, difíceis de discriminar). Isto sugere que, nestas condições, os recursos limitados do executivo central não puderam ser adequadamente distribuídos nas duas tarefas, dificultando que as ações fossem executadas apropriadamente, conforme os objetivos anteriormente estabelecidos. Neste caso, o sistema de armazenamento não foi suprimido como um todo, mas sim, houve interferência no mecanismo responsável por produzir e organizar a resposta em função dos objetivos (Szmalec, 2005).

A interação entre ordem de execução e instrução de prioridade também pode ser discutida em termos de objetivos da tarefa. A combinação entre estes fatores sugere que a memória para a localização dos estímulos incluiu a dimensão espacial, e também, uma representação das regras de instrução da tarefa. De acordo com esta concepção, a apresentação das instruções estabelece uma relação de variáveis (construção da regra) que é mantida e recuperada no momento da resposta. Uma vez estabelecida, esta regra pode ser recuperada mediante pouco esforço (Cohen-Kdoshay \& Meiran, 2007).

Este argumento pode explicar por que priorizar primeiro o tom, depois a localização contribuiu para que na segunda sessão (priorizar a localização, depois o tom) houvesse uma melhora significativa do desempenho espacial. Neste caso, supõe-se que, inicialmente, foi criada uma regra de execução com base em uma demanda complexa (responder ao espacial, sendo a discriminação auditiva a prioridade), que foi generalizada na realização da segunda sessão. Pode-se dizer que o conteúdo da memória (que na primeira sessão envolvia uma representação de uma regra complexa de execução) guiou a atenção para a codificação dos estímulos na segunda sessão e para a ativação de respostas específicas, facilitando o desempenho na tarefa (Cohen-Kdoshay \& Meiran, 2007). Estudos anteriores estabeleceram que informações mantidas na memória de trabalho guiam a atenção para o processa- 
mento posterior de estímulos relacionados àquele conteúdo memorizado (Soto \& Humphreys, 2009).

Além de codificar e manter a informação ativa na memória, o executivo central também coordena a realização de tarefas concomitantes. Mostramos que os recursos do executivo central foram distribuídos em duas tarefas, uma correspondente ao esboço visuoespacial e outra atentiva, mas de modalidade auditiva. Uma vez que o sistema é de capacidade limitada, as duas tarefas concorreram entre si por recursos, o que culminou na queda da capacidade tanto de armazenamento visuoespacial, quanto de discriminação de tons (Baddeley, 2002; Repovš \& Baddeley, 2006).

As considerações acerca de um sistema atentivo de capacidade limitada estão de acordo com o modelo do Sistema Atentivo Supervisor (Norman \& Shallice, 1986). Conforme este modelo a realização de tarefas complexas culmina na necessidade de execução de novos esquemas de resposta, diferentes dos esquemas usados para ações rotineiras. No presente estudo, esquemas diferentes ativaram as respostas para a tarefa de discriminação de tons e para a tarefa de localização espacial. Estes esquemas competiram pelos mesmos recursos de ativação, tendo como consequência um prejuízo no desempenho das tarefas.

Nossos dados concordam com estudos anteriores feitos no campo da neuropsicologia que indicaram como função do executivo central coordenar a realização de duas tarefas cognitivas de diferentes modalidades de estímulo. Pacientes com a doença de Alzheimer, por exemplo, têm dificuldades em desempenhar bem tarefas que são realizadas concomitantemente porque, nesta condição, a capacidade do executivo central de dividir a atenção entre os objetivos de resposta é excedida em virtude de lesões em regiões cerebrais envolvidas com este sistema (Logie, Cocchini, Della Sala \& Baddeley, 2004).

A interação entre similaridade dos tons, posição serial e instrução de prioridade confirma que recursos do executivo central foram direcionados para o sistema visuoespacial de armazenamento, entretanto, considera-se que estes recursos são limitados em termos de capacidade e de coordenação de recursos ao longo do tempo (Cowan, 2005). O desempenho da memória foi prejudicado porque os recursos atentivos foram saturados quando a capacidade máxima foi atingida ao aumentar a dificuldade da tarefa (tons de similaridade alta e prioridade auditiva). Este processo impediu a adequada distribuição de recursos para a atualização de informações na memória de trabalho, dificultando a recuperação do primeiro item apresentado.

Evidências de estudos anteriores com imagens cerebrais são compatíveis com a consideração de que o executivo central está envolvido com a função de atualizar as informações na memória de trabalho, ou seja, modificar continuamente uma representação na memória, na medida em que novos itens são acrescentados ao sistema. Quando a capacidade é excedida, há um prejuízo no desempenho da tarefa (Kiss, Pazderka-Robinson \& Floden, 2001).

Consideramos que recursos do executivo central estão envolvidos na codificação da informação visuoespacial na memória de trabalho. Recursos gerais interagiram com recursos específicos para criar uma representação visuoespacial e para mantê-la ativa, de modo a guiar as respostas segundo os objetivos da tarefa. Certamente estes recursos são limitados, o que torna a representação vulnerável às interferências como dificuldade da tarefa e quantidade de itens a serem memorizados.

\section{Referências}

Ang, S. Y., \& Lee, K. (2010). Exploring developmental differences in visual short-term memory and working memory. Developmental Psychology, 46(1), 279-285.

Baddeley, A. D. (2002). Fractionating the central executive. In D. T. Stuss \& R. T. Knight (Eds.), Principles of frontal lobe function (pp. 246-260). New York: Oxford University Press.

Baddeley, A. D. (2007). Working memory, thought, and action. New York: Oxford University Press.

Brooks, L. R. (1967). The suppression of visualization by reading. The Quarterly Journal of Experimental Psychology, 19(4), 289-299.

Cohen-Kdoshay, O., \& Meiran, N. (2007). The representation of instructions in working memory leads to autonomous response activation: Evidence from the first trials in the flanker paradigm. The Quarterly Journal of Experimental Psychology, 60(8), 1140-1154.

Collette, F., \& Van der Linden, M. (2002). Brain imaging of the central executive component of working memory. Neuroscience and Biobehavioral Reviews, 26, 105-125.

Cowan, N. (2005). Working memory capacity. New York: Taylor $\&$ Francis Group.

Curran T., \& Keele S. W. (1993). Attentional and nonattentional forms of sequence learning. Journal of Experimental Psychology: Learning, Memory, \& Cognition, 19, 189-202.

D'Esposito, M. (2007). From cognitive to neural models of working memory. Philosophical Transation of Royal Society. B, 362, 761-772.

Fisk, J. E., \& Sharp, C. A. (2003). The role of the executive system in visuo-spatial memory functioning. Brain and Cognition, 52, 364-381.

Frensch, P. A., Lin, J., \& Buchner, A. (1998). Learning versus behavioral expression of the learned: The effects of a secondary tone-counting task on implicit learning in the serial reaction time task. Psychological Research, 61, 83-98.

Galera, C., \& Fuhs, C. C. (2003). Memória visuoespacial a curto prazo: Os efeitos da supressão articulatória e de uma tarefa aritmética. Psicologia: Reflexão e Crítica, 16(2), 337-348.

Henson, R. N. A., Burgess, N., \& Frith, C. D. (2000). Recoding, storage, rehearsal and grouping in verbal short-term memory: An fMRI study. Neuropsychologia, 38, 426-440.

Jiang, Y., Olson, I. R., \& Chun, M. M. (2000). Organization of visual short-term memory. Journal of Experimental Psychology: Learning, Memory, and Cognition, 26(3), 683-702.

Kiss, I., Pazderka-Robinson, H., \& Floden, D. (2001). Eventrelated brain potentials and central executive function: Further evidence for Baddeley's model. Journal of Psychophysiology, $15,1-13$.

Klauer, K. C., \& Zhao, Z. (2004). Double dissociations in visual and spatial short-term memory. Journal of Experimental Psychology: General, 133(3), 355-381. 
Klauer, K. C., \& Stegmaier, R. (1997). Interference in immediate spatial memory: Shifts of spatial attention or central-executive involvement? The Quarterly Journal of Experimental Psychology, 50A(1), 79-99.

Logie, R. H. (1995). Visuo-spatial working memory. Hove: Lawrence Erlbaum.

Logie, R. H., Cocchini, G., Della Sala, S., \& Baddeley, A. D. (2004). Is there a specific executive capacity for dual task co-ordination? Evidence from Alzheimer's Disease. Neuropsychology, 18, 504-513.

Maybery, M. T., Clissa, P. J, Parmentier, F. B. R., Leung, D., Harsa, G., Fox, A. M., \& Jones, D. M. (2009). Binding of verbal and spatial features in auditory working memory. Journal of Memory and Language, 61(1), 112-133.

Miller, E. K., \& Cohen, J. D. (2001). An integrative theory of prefrontal cortex function. Annual Review of Neuroscience, 24, 167-202.

Miyake, A., Friedman, N. P., Rettinger, D. A., Shah, P., \& Hegarty, M. (2001). How are visuospatial working memory, executive functioning, and spatial abilities related? A latent-variable analysis. Journal of Experimental Psychology: General, 130(4), 621-540.

Nissen, M. J., \& Bullemer, P. (1987). Attentional requirements of learning: Evidence from performance measures. Cognitive Psychology, 19, 1-32.

Norman D. A., \& Shallice, T. (1986). Attention to action: Willed and automatic control of behaviour. In R. J. Davidson, G. E. Schwartz, D. Shapiro (Eds.), Consciousness and self-regulation (V. 4, pp. 1-18). New York: Plenum.

Olson, I. R., \& Marshuetz, C. (2005). Remembering “what” brings along "where" in visual working memory. Perception \& Psychophysics, 67, 185-194.

Repovš, G., \& Baddeley, A. (2006). The multi-component model of working memory: Explorations in experimental cognitive psychology. Neuroscience, 139, 5-21.
Rudkin, S. J., Pearson, D. G., \& Logie, R. H. (2007). Executive processes in visual and spatial working memory tasks. The Quarterly Journal of Experimental Psychology, 60(1), 79-100.

Salway, A. F., \& Logie, R. H. (1995). Visuospatial working memory, movement control and executive demands. British Journal of Psychology, 86(2), 253-269.

Schneider, W., Eschman, A., \& Zuccolotto, A. (2002). E-Prime reference guide. Pittsburgh: Psychology Software Tools Inc.

Soto, D., \& Humphreys, G. W. (2009). Automatic selection of irrelevant object features through working memory: Evidence for top-down attentional capture. Experimental Psychology, $56,165-172$.

Stadler, M. A. (1995). Role of attention in implicit learning. Journal of Experimental Psychology: Learning, Memory, and Cognition, 21, 819-827.

Szmalec, A. (2005). Functional unity and diversity within executive control: A study by means of parametrical variations of executive involvement in reaction time tasks. Tese de Doutorado, Universiteit Gent, Bélgica.

Walker, P., Hitch, G. J., \& Duroe, S. (1993). The effect of visual similarity on short-term memory for spatial location: Implications for the capacity of visual short-term memory. Acta Psychologica, 83, 203-224.

Recebido em 17.05.2010

Primeira decisão editorial em 28.06.2011

Versão final em 07.07.2011

Aceito em 14.07.2011 